\title{
Ancrage territorial du modèle contractuel rizicole sénégalais : une perspective géographique et relationnelle
}

\author{
Jean Marie Fodé Touré ${ }^{1,2,4,{ }^{*}}$, Pascal Chevalier ${ }^{1}$ (D) et Jérémy Bourgoin ${ }^{3,4}$ (D) \\ ${ }^{1}$ UR LAGAM - Université Paul Valéry Montpellier 3, Montpellier, France \\ 2 CIRAD, UMR INNOVATION, F-34398 Montpellier, France \\ 3 CIRAD, UMR TETIS, F-34398 Montpellier, France \\ ${ }^{4}$ Univ. Montpellier, Montpellier, France
}

\begin{abstract}
Résumé - Pour relever les défis de l'agriculture africaine, notamment de l'autosuffisance alimentaire, de la création d'emploi et de richesse, le modèle du contrat agricole est promu par certains États et bailleurs de fonds internationaux dans le cadre d'un discours performatif sur les vertus des partenariats entre agroindustries et producteurs. Toutefois, les contrats agricoles suscitent des interprétations contradictoires. Les uns en font un modèle qui aurait des effets d'entraînement sur l'économie rurale, tandis que les autres y voient des stratégies de prédation des ressources agricoles, qui sont néfastes pour les communautés locales. D'un côté comme de l'autre, les analyses restent centrées sur des questions éthiques et parfois idéologiques. Les conditions de fonctionnement des contrats et leurs impacts en termes de développement sont peu abordés. Notre contribution, basée sur des enquêtes sociologiques menées au Sénégal, vise à caractériser objectivement les formes d'interaction (positive ou négative) entre contrats agricoles et développement des territoires qui abritent une filière rizicole. C'est à partir de ces enjeux de croisement entre développement agricole et développement territorial que nous développons une démarche méthodologique permettant de réaliser ce type de diagnostic. À l'échelle des régions dominées par la riziculture, nous mettons en exergue des trajectoires territoriales contrastées par rapport à l'interaction entre modèle agricole et développement territorial. Ce diagnostic est interprété par la connaissance des systèmes d'acteurs, notamment à l'aide de deux indicateurs, à savoir: la centralité, qui donne une indication sur les questions de gouvernance territoriale, et la densité des liens, qui renseigne sur l'importance du contrat agricole par rapport à d'autres modes de mise en relation. Concernant l'insertion des contrats agricoles, la structure des réseaux d'acteurs permet d'identifier un mode de gouvernance centralisé autour de la banque agricole nationale et de quelques industriels. Mais on note aussi une persistance des rapports informels et des structures communautaires (familles, amis, voisins) qui fonctionnent en parallèle du contrat agricole et qui remettent en cause le discours politique par rapport au potentiel transformateur des contrats agricoles.
\end{abstract}

Mots clés : Sénégal / filière agricole / agriculture contractuelle / gouvernance des filières / riz

\begin{abstract}
Territorial embeddedness of the Senegalese rice value chain contractual model: geographical and relational perspectives. To meet the challenges of African agriculture, particularly food self-sufficiency, job creation and wealth, the agricultural contract model is being promoted by some governments and international donors in the framework of a performative discourse on the virtues of partnerships between agro-industries and producers. However, agricultural contracts give rise to contradictory interpretations. Some see it as a model that would have spillover effects on the rural economy, while others see it as a strategy of predation of agricultural resources, which is harmful to local communities. On both sides, analyses remain focused on ethical and sometimes ideological issues. The operating conditions of the contracts and their impacts in terms of development are little addressed. Our contribution, based on sociological surveys conducted in Senegal, aims at objectively characterizing the forms of interaction (positive or negative) between agricultural contracts and the development of the territories where the rice sector is located. On the basis of these issues of articulation between agricultural development and territorial development, we are proposing a methodological approach to carry out this type
\end{abstract}

\footnotetext{
$\overline{\text { *Auteur de correspondance }}$ : jmftoure@gmail.com
} 
of diagnosis. At the scale of the regions dominated by rice growing, we highlight that the territorial trajectories are contrasted in relation to the interaction between the agricultural model and territorial development. This diagnosis is interpreted through the knowledge of the systems of actors, in particular with the help of two indicators, namely centrality, which gives an indication of the issues of territorial governance, and the density of social relations, which provides information on the importance of the agricultural contract in relation to other modes of relationship building. With regard to the insertion of agricultural contracts, the structure of the networks of actors makes it possible to identify a centralized mode of governance at the level of the national agricultural bank and a few agro-industrial enterprises. Nevertheless, informal relationships and traditional local structures (families, friends, neighbors) persist and function in parallel with the agricultural contract, calling into question the political discourse regarding the transformative potential of agricultural contracts.

Keywords: Senegal / agricultural value chains / contract farming / value chain governance / rice

\section{Introduction}

En réponse aux enjeux d'autosuffisance alimentaire et de réduction de la pauvreté qui perdurent depuis les années 1960 dans certains pays d'Afrique de l'Ouest, les États concernés et leurs partenaires techniques et financiers promeuvent dorénavant des partenariats entre producteurs et industriels. Les objectifs visés sont l'amélioration de la productivité agricole et plus généralement l'accompagnement du développement rural (Babu et al., 2015). Cependant, ces modèles de partenariat, qui sont répliqués dans la plupart des pays africains, suscitent la controverse.

Ce débat est en partie animé par des États africains, des grandes firmes, des bailleurs de fonds et des organisations internationales qui soutiennent la thèse selon laquelle le modèle agricole promu aurait des effets d'entraînement bénéfiques sur les populations locales en termes de création de richesses et d'emploi. Ce groupe d'acteurs considère ces effets comme allant de soi et n'explicite ni les mécanismes de cet entraînement ni les conditions de mise en œuvre pour qu'ils se produisent effectivement (Gabas et al., 2019). Les autres protagonistes du débat, les organisations paysannes et les ONG, sont critiques vis-à-vis des modèles agricoles en général, et notamment ceux importés par l'État, et vis-à-vis des appuis externes au monde agricole, qui paraissent le plus souvent inadaptés. La réussite des contrats agricoles est analysée par plusieurs chercheurs (Prowse, 2013) qui mobilisent la théorie des coûts de transaction, laquelle précise les conditions sous lesquelles ce mécanisme est bénéfique pour les producteurs et pour les investisseurs. Une des conditions de réussite du contrat est la spécificité du produit. En effet, les denrées hautement périssables, difficiles à cultiver ou à haute valeur par unité de poids s'échangent généralement par le mécanisme du contrat (Minot, 2011). Cependant, des travaux empiriques ont démontré que des produits considérés comme non adaptés au contrat (par exemple, le riz), sont pourtant échangés par ce mécanisme, notamment grâce aux conditions spécifiques du lieu concerné. Il s'agit des conditions suivantes (GIZ, 2014) : (i) le déficit d'infrastructures et de systèmes de transport pouvant créer des opportunités; (ii) la bonne collaboration dans le passé entre producteurs et acheteurs; (iii) le regroupement des producteurs au sein d'organisations paysannes opérationnelles; (iv) les investissements qui lient des entreprises et des producteurs et qui créent une forte interdépendance; (v) le sens éthique des entreprises qui sont capables de, et motivées à, investir dans les projets de développement communautaire et; (vi) des conditions cadres propices en matière de politiques, de législation et de respect des contrats, ainsi que des subventions adaptées et un accès aux services d'appui qui renforcent l'engagement à respecter les contrats.

L'émergence et le développement de ces contrats sont considérés par les économistes et les gestionnaires comme des innovations organisationnelles pour assurer une meilleure coordination dans les filières (Faure et al., 2018). En contrepartie, le contrat génère également des coûts et, à un moment donné, il devient moins efficace que le marché ou l'intégration hiérarchique. Toutefois, le problème est de savoir identifier ce moment. Les conditions de réussite abordées cidessus donnent une idée de la complexité des facteurs de réussite des contrats. Pourtant, de nombreux acteurs sont favorables à cette pratique, qu'ils considèrent comme devant devenir une norme du fait de son potentiel de contribution à une croissance socio-économique inclusive. La contractualisation dans les filières domestiques comporterait un réel potentiel en termes de réduction de la pauvreté puisque ces filières concentrent un nombre important de petits producteurs qui sont majoritairement pauvres. C'est une hypothèse forte de la littérature sur les contrats agricoles, mais sur laquelle les avis sont partagés (Prowse, 2013). Dans tous les cas, on note un lien entre les contrats agricoles et la réduction de la pauvreté dans la mesure où les petits producteurs dépensent leurs revenus localement, ce qui a un effet démultiplicateur (Hazell et al., 2006).

Dans le contexte spécifique du Sénégal, les résultats des premières expériences dans la filière rizicole sont controversés. Certaines causes identifiées portent sur le non-respect des contrats (Diouf, 2014). En effet, le non-respect des contrats est une contrainte majeure qui s'explique par la rigidité des contrats dans un environnement instable. Le contrat fixe des conditions de prix avant la récolte en fonction des prévisions de rendement moyen que les producteurs ne maîtrisent pas, en raison des nombreux facteurs qui sont instables (pluviométrie, accès aux intrants, etc.). Les rendements atteints sont souvent en deçà des prévisions, poussant les producteurs à se désengager de contrats qui ne sont plus rémunérateurs. Pour y remédier, il faudrait intégrer plus de flexibilité dans les contrats. Il faudrait, également, favoriser la mise en place de cadre multi-acteurs pour réfléchir à une meilleure valorisation des productions, un partage plus équitable de la valeur et la mobilisation d'organisations de producteurs compétentes et capables d'exercer un pouvoir de marché (FARM, 2018). 
Les contrats agricoles ont vocation à transformer un espace géographique par des nouvelles formes d'organisations. Nous distinguons la construction des contrats par les pouvoirs publics, par les acteurs locaux et par les gestionnaires du territoire. En effet, nous considérons que le territoire est un construit social qui s'appuie sur des interactions historiques d'acteurs. Et que c'est à une échelle micro qu'il est possible d'analyser de manière fine ces relations sociales. Ainsi, il s'agit de comprendre l'ancrage territorial des modèles contractuels pour expliquer comment, à certains endroits, la logique contractuelle s'articule de manière positive avec le territoire, tandis qu'à d'autres, l'ancrage est plus difficile à réaliser.

Selon notre hypothèse, l'ancrage va dépendre de trois dimensions: (i) le contexte (gouvernance, ressources locales, etc.); (ii) le système d'acteurs (moyens d'actions) et; (iii) la structure de marché, selon qu'il est de type concurrentiel, monopsone (un demandeur et un grand nombre d'offreurs) ou oligopole (de nombreux demandeurs et quelques offreurs). La logique contractuelle s'articule mieux dans une structure de marché de type monopsone où les stratégies opportunistes ne s'expriment guère, contrairement au cas du marché concurrentiel (Prowse, 2013).

Le contrat agricole, défini comme un accord écrit, représente une part minime de l'ensemble des liens existant en milieu rural sénégalais. Toutefois, ce type particulier permet d'avoir à des preuves de l'existence d'une relation. En effet, le contrat n'est pas interprété, il permet uniquement de confirmer l'existence d'un lien, qui est ensuite analysé pour vérifier s'il est en capacité d'être appliqué et respecté par les parties.

\section{Méthodes}

\subsection{Contexte et ancrage du modèle contractuel rizicole au Sénégal}

À partir du début du vingtième siècle, la colonisation française a importé et imposé le riz au Sénégal pour couvrir les besoins alimentaires des consommateurs urbains et de la maind'œuvre agricole tout en libérant des terres, anciennement dédiées aux cultures vivrières, pour la culture industrielle de l'arachide (Bonnefond et Couty, 1988). À l'Indépendance, en 1960, le gouvernement sénégalais a continué d'investir dans la filière rizicole dans une perspective d'autosuffisance alimentaire nationale et donc de réduction de la dépendance du pays vis-à-vis de l'extérieur (Bélières et Touré, 1999). Cinquante années plus tard, ces enjeux sont toujours d'actualité, puisque le riz est plus que jamais une denrée stratégique pour le Sénégal compte tenu de son importance dans la consommation des ménages et de son poids dans la balance commerciale. Cependant, la production nationale ne couvre que $35 \%$ des besoins du pays, estimés entre 1,8 et 1,9 million de tonnes en équivalent riz blanc (Mendez del Villar, 2019). Le soutien à la filière domestique est donc une problématique de taille qui interpelle fortement les pouvoirs publics, surtout avec la récurrence des chocs exogènes tels que la crise alimentaire de 2008.

À l'échelle locale, on constate des déséquilibres territoriaux et des zones de production rizicole hétérogènes reflétant des choix politiques, des potentiels et des enjeux particuliers à chaque territoire. Dès 1960, les investissements publics se sont concentrés dans le département de Dagana (delta du fleuve Sénégal), identifié comme une zone à forte potentialité agricole pour atteindre l'autosuffisance nationale en riz. Dans les années 1970-1980 (par suite des périodes de sécheresse), les interventions sur le riz se sont étendues à d'autres zones suivant une logique différente, car dans une perspective d'autosuffisance alimentaire au niveau local (cas du département de Podor). Aujourd'hui, le département de Dagana se détache à tous les niveaux de la filière (production, transformation, services d'appui), comparé aux autres principales zones de production de riz irrigué au Sénégal, à savoir les départements de Podor et de Vélingara (bassin de l'Anambé) (Fig. 1). Ces deux dernières sont relativement proches en termes de filière rizicole, étant confrontées à l'insuffisance des aménagements hydro-agricoles et à la faible présence des opérateurs de la filière au niveau départemental. Néanmoins, certains s'interrogent sur les performances relativement faibles de la filière selon les zones par rapport aux investissements consentis (Fall, 2015).

Ces différences d'intégration entre filière rizicole et territoire permettent de justifier le choix des zones d'étude. Il s'agit des deux départements les plus contrastés en termes de développement de la filière rizicole localisée: Dagana et Vélingara. Dagana est considéré par le gouvernement du Sénégal et certains bailleurs de fonds comme un idéal par rapport aux conditions favorables de la filière et du territoire, comparé à Vélingara qui essayerait de suivre cette trajectoire et de tendre vers ce modèle. Ces contrastes sont pertinents pour mettre en exergue des effets de contexte et des systèmes d'acteurs dans les processus d'ancrage territorial du modèle contractuel.

Le modèle des contrats agricoles est, au Sénégal, une innovation qui a pour objectif d'influencer le développement territorial. Dans ce cadre, deux formes sont promues par l'État et certains bailleurs de fonds, avec l'hypothèse qu'elles auraient des effets structurants sur les territoires. Il s'agit d'abord du modèle mixte, matérialisé par un contrat tripartite impliquant producteurs rizicoles, opérateurs privés et institutions parapubliques (appui technique, financement). Au Sénégal, ce modèle est structuré par la banque agricole d'État qui accorde un crédit de campagne au producteur à condition qu'il accepte de rembourser en nature dans le cadre d'un second contrat entre la banque et un industriel rizier. Il s'agit ensuite du modèle privé, caractérisé par des relations contractuelles structurées par des coopératives ou des entreprises dans lesquelles sont impliquées des producteurs. Dans nos zones d'étude, cette forme se nomme contrat de préfinancement et met en lien une agro-industrie et des producteurs pour le financement des intrants agricoles en échange d'un remboursement en nature ou en espèces. Généralement, les producteurs qui se tournent vers cette forme de contractualisation n'ont pas accès au crédit de la banque agricole. Cette dernière ne finance pas toujours les producteurs détenant moins de 5 ha ni ceux qui sont en situation d'endettement. Ces deux formes de contractualisation sont érigées en modèle de réussite par le gouvernement du Sénégal et certains bailleurs de fonds. Toutefois, l'existence du territoire est bien antérieure à ces contrats et fonctionne sur d'autres modes de coordination, qui s'appuient notamment sur des relations de confiance. 


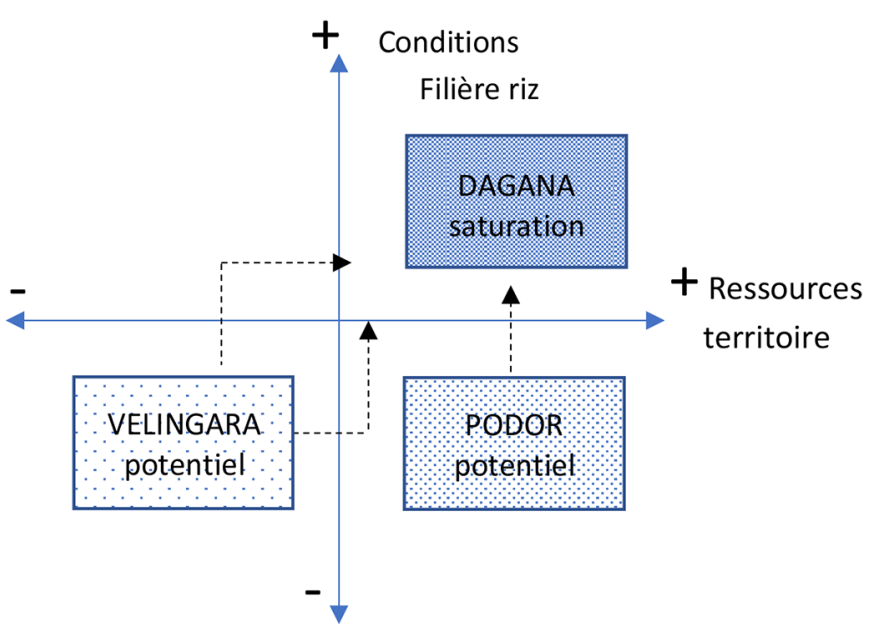

Fig. 1. Trois situations d'interaction entre filière rizicole et territoire. Fig. 1. Three situations of interaction between the rice sector and the territory.

Comment ces formes s'articulent-elles donc avec le territoire?

\subsection{Approche par les relations sociales pour mesurer l'ancrage}

Les acteurs façonnent les activités et se donnent les moyens d'agir sur le territoire en s'appuyant sur les relations sociales. Nous proposons de reconstituer les structures relationnelles avec l'aide des outils de la sociologie des réseaux sociaux (Lazega, 2014). Il s'agit d'un cadre d'analyse des macrostructures de réseau qui repose sur une conception simple de trois fondamentaux caractérisant un réseau: des nœuds (acteurs) incluant leurs caractéristiques; des liens incluant leur nature, force et polyvalence; des propriétés structurelles, dont cinq principales qui permettent de caractériser la nature des changements au sein des systèmes d'acteurs (Ahuja et al., 2012).

Nous avons identifié, dans chacun des deux départements rizicoles, un échantillon de producteurs qui appartiennent à un même périmètre irrigué et qui bénéficient d'un dispositif de contrat agricole (Fig. 2). Ce choix s'inscrit dans la branche des réseaux complets qui consiste à définir au préalable les frontières d'un ensemble social donné avant de reconstituer, dans ce dernier, les relations sociales. Deux groupes d'acteurs sont analysés. Le premier est localisé dans le département de Dagana, au niveau d'un périmètre aménagé nommé «3PRD» (Projet de promotion du partenariat rizicole dans le Delta, zone de la Société nationale d'aménagement et d'exploitation des terres du delta du fleuve Sénégal et des vallées du fleuve Sénégal et de la Falémé-SAED) et compte 145 producteurs attributaires qui se répartissent sur 2325 ha aménagés en un seul tenant. Le second groupe est situé dans le département de Vélingara, dans un périmètre nommé « secteur G » (zone de la Société de développement agricole et industriel du Sénégal-SODAGRI) qui compte environ 130 producteurs qui se partagent 1180 ha aménagés en un seul tenant. Ces deux périmètres irrigués ont été étudiés à des

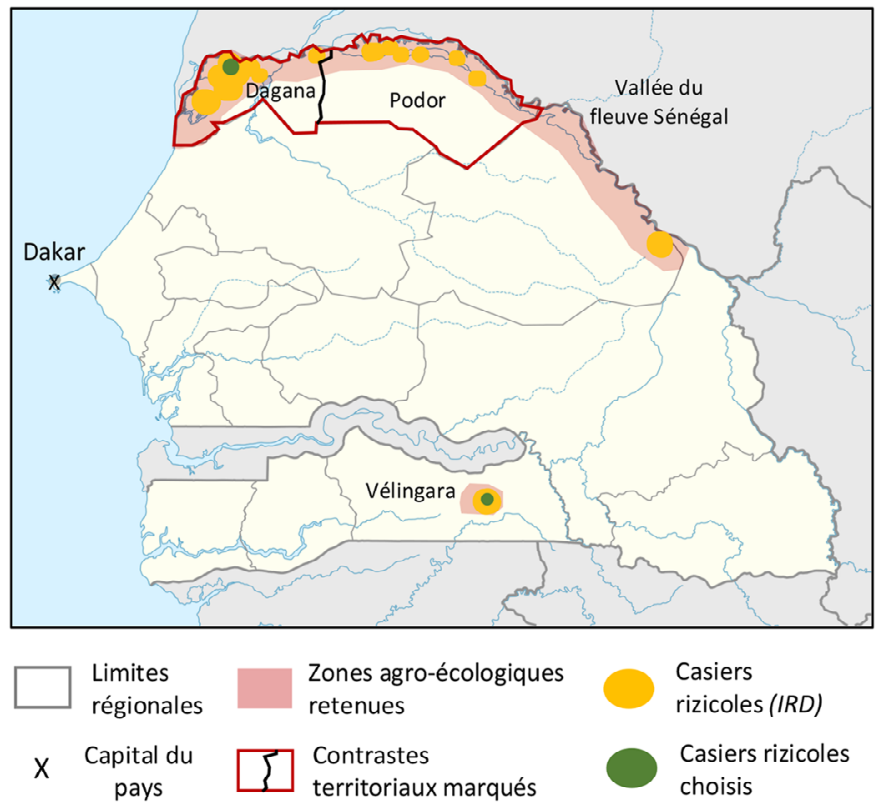

Fig. 2. Localisation des zones d'études et des groupes de producteurs étudiés.

Fig. 2. Location of study area and producers' groups surveyed.

moments différents par rapport au processus de construction d'une ressource territoriale, qui comprend plusieurs phases (création du foncier irrigué, développement, dégradation et réhabilitation). Le périmètre du $3 \mathrm{PRD}$ aménagé en 1990-1991 par l'Union des Groupements d'intérêt économique du Delta (UGIED) sur financement privé, entre dans un nouveau cycle après la réhabilitation du casier irrigué dans le cadre du Projet de promotion des partenariats rizicoles dans le Delta (2011-2019) visant à promouvoir un nouveau modèle d'aménagement des terres reposant sur des investissements à coûts partagés entre l'État et les producteurs. En comparaison, le périmètre Secteur G (aménagé en 1996-1999 par la SODAGRI sur financement public) est à la fin de son premier cycle et attend (depuis 2018) d'être réhabilité dans le cadre du Projet Tiers-Sud, car actuellement seulement la moitié des superficies est exploitable (soit environ 650 sur 1180 ha), à cause de la vétusté des aménagements hydro-agricoles. Toutefois, la réhabilitation a pris beaucoup de retard et est toujours en cours (août 2021). Selon le ministre de l'Agriculture, le secteur $\mathrm{G}$ sera réhabilité au plus tard le $1^{\text {er }}$ juillet 2022. Le choix de ces deux périmètres spécifiques est intéressant dans la mesure où c'est le même bailleur de fonds (l'Agence française de développement) qui appuie la structuration des deux périmètres et il est fort probable que le modèle utilisé dans le périmètre du 3PRD sera transféré dans celui du Secteur G. Cependant, selon notre hypothèse, le transfert du modèle contractuel ne va pas donner les mêmes résultats partout et des particularités vont émerger, de manière plus ou moins forte, en fonction des effets de contextes territoriaux.

Après le choix des groupes de producteurs, nous avons fixé la taille de l'échantillon en fonction du nombre d'enquêtes envisageables par rapport à la durée de la mission sur le terrain (10 jours par zone d'étude), soit 35 entretiens par zone d'étude. 
Ainsi, nous avons construit un échantillonnage situationnel qui s'appuie sur des variables qualitatives, selon la possession par les producteurs de ressources tangibles (matériel agricole, semences) et intangibles (statut social, responsabilité politique). Ce choix se fonde sur l'hypothèse selon laquelle le statut de l'acteur déterminerait sa place au sein du périmètre. Néanmoins, cette hypothèse est mise à l'épreuve via l'analyse croisée des données relationnelles avec celles des statuts d'acteurs. Par ailleurs, pour éviter le biais de sélection de l'échantillonnage, nous avons ouvert le réseau social en dehors de l'échantillon et du périmètre. Cela permet de nuancer la structuration du réseau social à l'intérieur du périmètre, tout en mettant en exergue des relations entre ce dernier et le territoire.

Pour reconstituer le réseau social, nous avons utilisé des générateurs de noms. Il s'agit de trois questions sociométriques qui correspondent à trois niveaux d'intensité des relations sociales, à savoir: les liens d'interconnaissance (personnes connues), les liens d'interdépendance (personnes actives) et les liens de conseil (personnes ressources). Une fois les enquêtes réalisées, les données relationnelles sont introduites dans le logiciel NodeXL ${ }^{\odot}$ qui permet de calculer les principales propriétés structurelles des réseaux et de donner des indications sur les conditions territoriales de la contractualisation agricole. À partir de deux métriques d'un réseau, la centralité et la densité des liens, nous renseignons les trois déterminants de l'ancrage territorial du modèle contractuel : le système de gouvernance, le système d'acteur et les ressources locales (Camagni, 2013).

La centralité désigne le sommet (acteur) ayant le plus de liens par lequel passe le plus de chemins et qui est le plus proche de tous les autres. À l'échelle individuelle, cet indicateur traduit la "popularité» de l'acteur et son niveau d'intégration dans le groupe. À l'échelle du groupe, la centralité donne une indication sur le niveau de hiérarchie (fort/faible), sur la distribution du pouvoir et donc sur le profil du système de gouvernance.

La densité correspond au nombre de liens existants par rapport au nombre de liens possibles entre tous les acteurs. Dans la mesure où les liens sont animés par des échanges de ressources (matérielles comme immatérielles), la densité du réseau donnera une indication quantitative de degré de coopération en son sein. Si la densité relationnelle n'est pas un indicateur de performance d'un système régional, elle reste un indicateur pertinent de la manière dont s'articulent concurrence et coopération dans une même région. Si de fortes densités semblent peu réalistes compte tenu des arbitrages que font les acteurs entre appropriation et partage des ressources, de faibles densités peuvent être, à l'inverse, le signe d'un manque d'ancrages locaux des acteurs et d'une fragilité du système régional (Vicente et al., 2018).

Les producteurs considérés dans cette étude ont tous bénéficié d'une parcelle de terre via un contrat agricole. Il ne s'agit pas ici de remettre en question ce contrat, mais plutôt d'analyser comment les producteurs développent des réseaux de contractualisation pour la mise en valeur de leur parcelle et la réussite du contrat sur la terre. L'analyse prend en compte la dimension géographique, c'est-à-dire des contrats agricoles situés géographiquement sur des territoires spécifiques que l'on compare. L'objectif n'est pas de comparer les contextes mais plutôt d'analyser le fonctionnement des contrats tout en mettant en exergue les éléments de contexte qui influencent ces processus.

\section{Résultats et discussions}

\subsection{Profil de la gouvernance territoriale du modèle contractuel}

Les structures décentralisées (ici la SAED et la SODAGRI) ont un rôle important à jouer dans le développement de l'irrigation au Sénégal. Ces sociétés publiques assurent deux principales missions, qui leur sont confiées par l'État: (i) la maîtrise d'ouvrage déléguée pour la réalisation des aménagements hydro-agricoles incluant les services de maintenance et de gestion de l'eau, et (ii) la fonction de conseil-appui auprès des communautés locales concernées par l'agriculture irriguée (Bazile et al., 2016). Toutefois, nos résultats montrent que sur certaines activités qui interviennent après la mise à disposition des parcelles aménagées, notamment l'accès aux intrants, au crédit et la mise en marché, c'est la banque agricole d'État qui apparaît comme un «acteur central» dans les deux groupes de producteurs étudiés, même si, dans l'Anambé, elle s'appuie sur une forte intermédiarité des organisations paysannes. Les autres institutions publiques, telles que les sociétés nationales d'aménagement des terres et les acteurs de la recherche agricole, sont plutôt à la périphérie, voire à la marge (Fig. 3). Pour comprendre ce résultat, il est important de rappeler le désengagement de l'État de l'appui au secteur agricole dans le cadre des Politiques d'ajustements structurels (PAS) des années 1980. Ce désengagement a fortement impacté ces structures décentralisées avec une réduction de leurs budgets et de leurs ressources humaines. Elles sont devenues dépendantes des appui externes (Naudet, 1999), et ont aussi été déstabilisées par une forte politisation à l'intérieur des institutions publiques (Haussaire, 2019). Pourtant, nous remarquons que la société nationale d'aménagement des terres a une place plus importante dans l'Anambé comparée à son homologue dans le département de Dagana. C'est la conséquence d'un désengagement de l'État intervenu plus tardivement dans l'Anambé, à la fin des années 1990, soit une décennie après le transfert de responsabilité effectué dans la zone de Dagana.

Pour continuer à faire fonctionner les filières stratégiques après le désengagement, plusieurs gouvernements africains ont proposé et accompagné la mise en place d'interprofessions. C'est le cas de la fédération des producteurs de l'Anambé, créée avec l'appui de l'État, qui assure l'interface entre les producteurs de la zone et la société nationale d'aménagement des terres, ainsi qu'avec les autres structures d'appui au secteur rural. À la différence de ce qui s'est passé en Europe, les Organisations de producteurs $(\mathrm{OP})$ ne résultent pas à l'origine d'une initiative indépendante et autonome des acteurs, mais plutôt d'une impulsion par des structures étatiques ou des partenaires extérieurs (États, ONG, partenaires techniques et financiers). Ces derniers désirent structurer et organiser les marchés en s'appuyant sur des OP. En effet, les OP peuvent jouer un rôle important dans la promotion des filières agricoles. Elles favorisent le dialogue entre les acteurs de ces filières et représentent le premier interlocuteur de l'État. Selon leur niveau de fonctionnement, elles assurent aussi la veille sur les réglementations agricoles et commerciales, et encouragent 


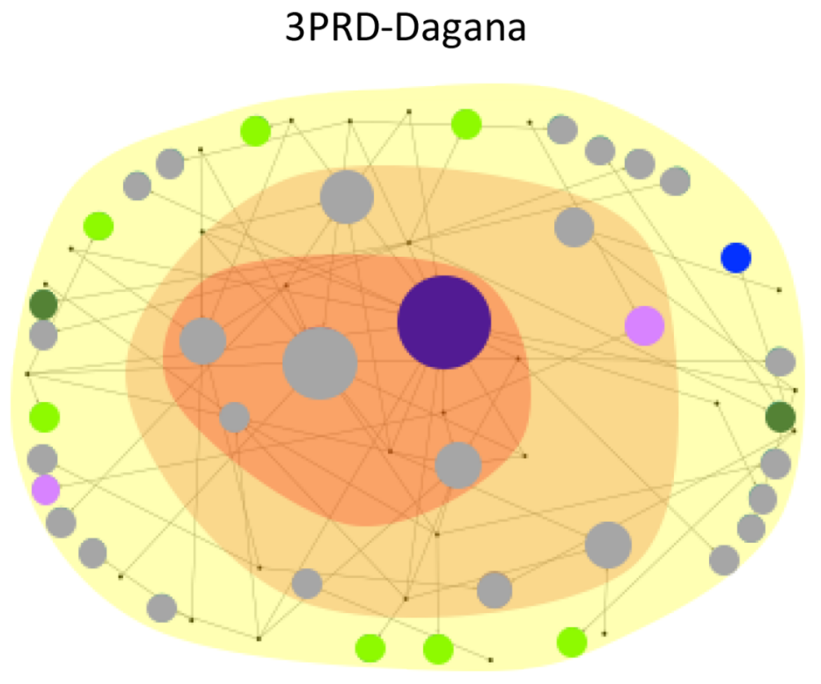

3PRD-Dagana

Position acteurs

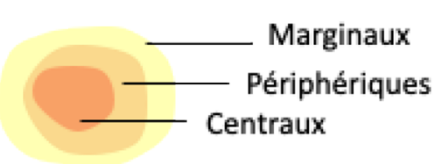

Statuts acteurs

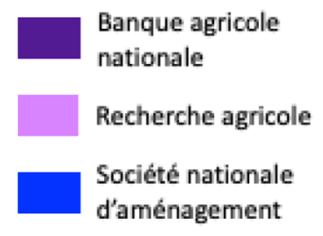

d’aménagement
Secteur G-Vélingara

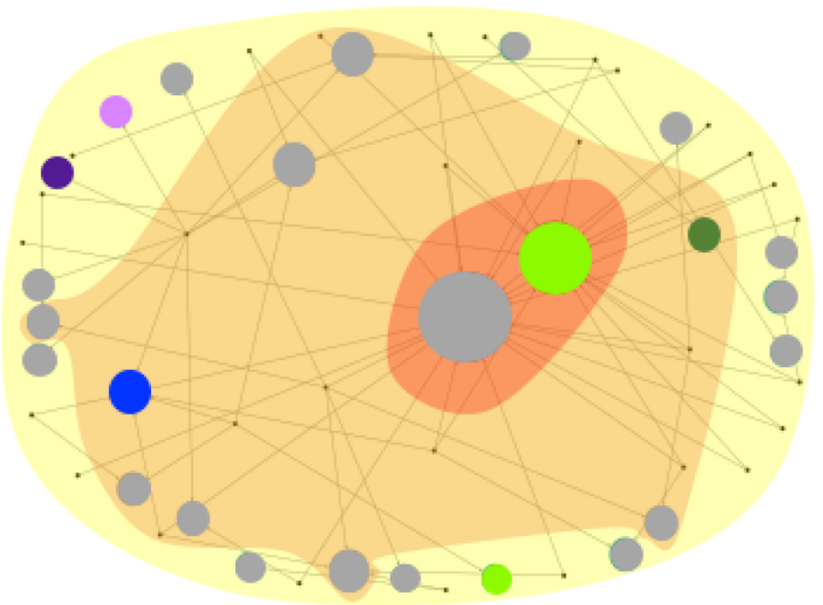

\section{Nombre de citation}

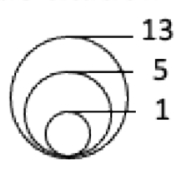
nationales

Filière riz (producteurs, prestataires, etc.)

Bailleurs de fonds

\section{paysannes locales et \\ Organisations}

Fig. 3. Positions des structures décentralisées par rapports aux groupes de producteurs.

Fig. 3. Positions of decentralized structures in relation to producers' groups.

l'amélioration de la qualité des produits, entre autres (Inter-réseaux et Lares, 2019). Mais la difficulté majeure réside dans le financement des OP et la forte influence des acteurs externes.

À côté de la banque agricole d'État, soulignons la place centrale de certains acteurs privés. Il s'agit des organisations de producteurs et des exploitations de taille moyenne (entre 5 et $25 \mathrm{ha}$ ). Toutefois, ce résultat contraste avec la vision de certaines ONG et organisations paysannes sur le rôle des élites nationales ou des acteurs internationaux qui serait important dans les groupes de producteurs. Ainsi, la centralité des acteurs ne s'explique pas uniquement par la possession de ressources tangibles (foncier irrigué, matériel agricole, etc.), mais aussi de ressources intangibles (responsabilités associatives et politiques). La ressource tangible relèverait du registre du «possessif» pouvant traduire des positions de pouvoir, tandis que la ressource intangible relèverait plutôt du registre du «représentatif» pouvant illustrer la proximité et la confiance. Ces deux types de ressources sont complémentaires pour expliquer la centralité. Cependant, dans certains périmètres rizicoles, l'un des deux types peut être plus important que l'autre dans la centralité. Par conséquent, la combinaison n'est pas toujours la même selon les territoires. Par exemple, ce n'est pas suffisant, dans un groupe de producteurs où il y a beaucoup de ressources tangibles réparties entre les membres (périmètre du 3PRD, Dagana), de n'avoir que des ressources intangibles pour être placé au cœur du groupe. Il faudrait avoir des ressources intangibles plus, à minima, une à deux ressources tangibles pour pouvoir avoir cette centralité. En comparaison, dans le périmètre du secteur $\mathrm{G}$ (Anambé), la configuration étant différente, avec moins de ressources tangibles, le fait d'avoir un minimum de ressources intangibles suffit pour être au cœur du groupe, à condition d'avoir facilement accès aux ressources tangibles. En fonction des deux configurations, l'une peut être suffisante. Mais dans tous les cas, la ressource intangible est indispensable. La gouvernance privée s'appuie donc fortement sur les structures locales et communautaires.

\subsection{Densité des coopérations dans les contrats agricoles}

Il est important de caractériser les liens sociaux afin de confirmer l'existence (ou pas) d'un réseau social et de coopérations économiques. Dans les deux groupes de producteurs, nous observons que les liens d'interconnaissances et de conseils ne sont pas corrélés avec les liens de coopérations. Pour plus d'interconnaissances et d'entraide dans le secteur G (Vélingara), nous avons en comparaison moins de coopérations dans la situation du périmètre 3PRD (Dagana) (Fig. 4). Ce résultat pourrait s'expliquer par la présence d'un système d'acteurs (sociétés nationales d'aménagement des terres, collectivités locales, acteurs privés et acteurs associatifs) bien organisé qui arrive à créer de la confiance et de la coopération malgré des degrés d'interconnaissance moindres, comme c'est le cas dans certains pays d'Europe centrale (Lacquement et Chevalier, 2016). Dans nos sites d'étude au Sénégal, le niveau relativement faible des coopérations, comparé à l'interconnaissance, est un révélateur 

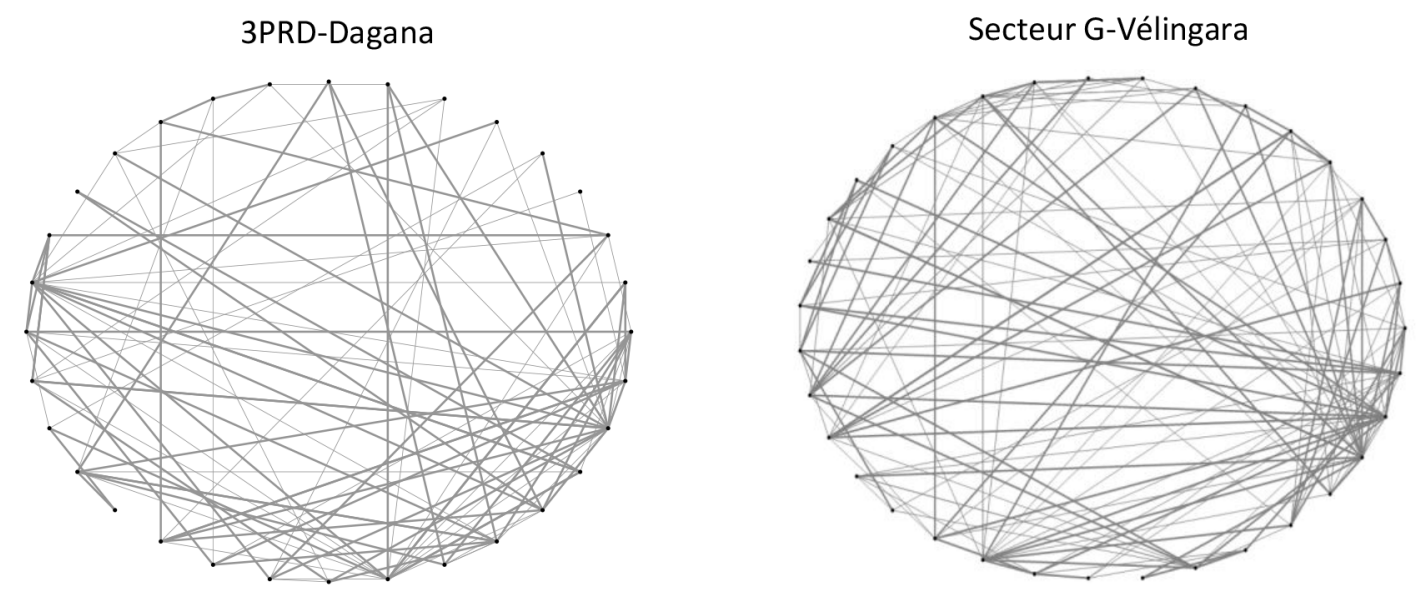

\begin{tabular}{|c|c|c|c|c|}
\multicolumn{2}{c|}{} & \multicolumn{3}{c}{$\begin{array}{c}\text { Liens de connaissance et de conseil } \\
\text { Liens de coopération }\end{array}$} \\
\hline \multirow{2}{*}{ Indicateur } & Liens de connaissance et de conseil & \multicolumn{2}{c|}{ Liens de coopération } \\
\cline { 2 - 5 } & 3PRD-Dagana & Secteur G-Vélingara & 3PRD-Dagana & Secteur G-Vélingara \\
\hline Densité des liens & 0,20 & 0,23 & 0,13 & 0,11 \\
\hline
\end{tabular}

Fig. 4. Densité des relations sociales et de coopération.

Fig. 4. Density of social relations and cooperation.

de la fragilité du système d'acteurs et donc d'un climat de méfiance vis-à-vis des institutions du marché et de l'État. Cette méfiance, qui est plus forte dans le groupe de l'Anambé que dans le groupe de Dagana, pourrait s'expliquer par trois hypothèses: (i) la faiblesse des ressources locales, la répartition déséquilibrée du foncier irrigué et le manque de transparence dans la gestion des terres irriguées du bassin de l'Anambé; (ii) le décalage temporel dans la dynamique d'insertion des contrats, qui est plus ancienne dans le département de Dagana, en postulant l'importance du temps (pour l'apprentissage) dans l'instauration de la confiance dans le contrat agricole; (iii) un service de conseil-appui qui ne répond pas aux attentes des producteurs et qui est absent de l'accès au crédit et de la mise en marché pour les producteurs de l'Anambé, contrairement au cas de leurs homologues de Dagana (Cissé et Diouf, 2015). En effet, un des facteurs de réussite des contrats dans le périmètre du 3PRD de Dagana, c'est l'association d'une diversité d'acteurs, dont les centres de gestion associatifs qui apportent des conseils-appuis dans la gestion comptable, administrative et financière (Hofmann et Papazian, 2020).

Nous observons donc un modèle de contrat agricole avec des acteurs centraux, dans l'offre de crédit à court terme à l'agriculture, qui sont la banque agricole d'État et certains acteurs privés, dont l'ambition est de renforcer les coopérations. Toutefois, les offres de crédit à moyen et long terme et les contrats portant sur l'achat de matériel agricole, l'aménagement ou la réhabilitation de parcelles irriguées sont rares.

\subsection{Place des réseaux informels}

Au Sénégal, en parallèle des contrats formels, il existe un réseau informel (famille, amis, voisins) qui fonctionne, et les producteurs font jouer la complémentarité entre les deux types de réseaux pour accéder aux ressources (semences, matériels agricoles, riz paddy, conseils agricoles, etc.). Nous pouvons affirmer la présence « de l'informel dans le formel » et révéler des failles du système formel. Par exemple, un producteur qui a besoin de garanties pour signer un contrat formel avec la banque agricole peut passer un accord informel avec un agroindustriel pour accéder au crédit de campagne. Autre exemple, un industriel peut solliciter du crédit au niveau des banques et ensuite financer les intrants de producteurs qui étaient pourtant exclus du système bancaire. Le système informel persiste donc, car les incitations à la formalisation sont faibles et le système formel ne couvre pas tous les besoins en financement.

\section{Conclusion}

Les contrats fonctionnent ici sur un modèle multipartite structuré par la banque agricole d'État, quelques privés et des organisations de producteurs. L'analyse des liens révèle une fragilité du système relationnel, dans lequel les liens d'interconnaissance sont relativement plus denses que les coopérations et donc, les taux de contractualisation portant sur des échanges de ressources (semences, matériel agricole, crédits de campagne agricole, riz paddy) sont relativement faibles. La majorité des producteurs développent un faible nombre de coopérations et l'essentiel de ces liens d'interdépendance porte sur de l'entraide et des échanges de service pour de la supervision de parcelle et passant par des réseaux informels (familles, amis et voisins). Ce résultat s'explique par un problème de méfiance vis-à-vis du système d'acteurs, et notamment des institutions du marché et de l'État.

Par conséquent, il est important de renforcer la confiance, en s'appuyant sur le rôle de la proximité. En effet, la proximité permet de tisser des liens de confiance sur le long terme. Par exemple, les bonnes relations entretenues depuis 1992 par la 
société familiale Danaya Céréales avec les organisations de producteurs du Mali lui assurent un approvisionnement régulier en matières premières et lui permettent de développer une filière fonio de qualité (FARM, 2018). En achetant les productions à un prix rémunérateur, en proposant aux producteurs divers services (conseil technique, encadrement, formation), mais aussi en investissant dans le développement local, l'acheteur suscite la confiance des communautés rurales et fidélise ses fournisseurs. Les entreprises doivent aussi s'appuyer sur un personnel technique solide et expérimenté, capable de diffuser des pratiques efficaces (FARM, 2018).

Pour créer de la confiance entre les différents maillons des filières, le contrat agricole doit également démontrer sa capacité à créer de la valeur et à la partager. La certification, qui garantit le respect de normes sociales et environnementales, est une voie prometteuse, même si la multiplication des labels est source de confusion. Travailler avec des OP peut être un atout, à condition que ces dernières soient compétentes et rendent de réels services. Enfin, les contrats gagneraient à être plus flexibles pour s'adapter aux fluctuations des marchés et permettre aux parties de gérer les risques (FARM, 2018).

Cet article permet de mettre en avant l'analyse des réseaux comme un des moyens de comprendre le fonctionnement du système contractuel et son ancrage territorial. Malgré les limites de la méthode (échantillonnage) et la possibilité d'avoir pu ignorer certains sous-systèmes d'acteurs de densité relationnelle plus importante à l'intérieur des périmètres, cet outil permet tout de même de mettre en lumière certaines questions et de faire quelques propositions pour guider l'action publique.

\section{Références}

Ahuja G, Soda G, Zaheer A. 2012. The genesis and dynamics of organizational networks. Organization Science 23(2): 34-448. https://doi.org/10.1287/orsc.1110.0695.

Babu SC, Manvatkar R, Kolavalli S. 2015. Strengthening capacity for agribusiness development and management in Sub-Saharan Africa. Africa Journal of Management 2(1): 1-30. https://doi.org/10.1080/ 23322373.2015.1112714.

Bazile AC, Vennat B, Dressayre E. 2016. Diagnostic institutionnel spécifique de la société de développement agricole et industriel du Sénégal (SODAGRI). Rapport d'études. COSTEA, 12 p. https:// www.comite-costea.fr/production/diagnostic-institutionnel-speci fique-de-la-societe-de-developpement-agricole-et-industriel-dusenegal-sodagri/.

Bélières JF, Touré HA. 1999. Impact de l'ajustement structurel sur l'agriculture irriguée du Delta du Sénégal. Thèse de doctorat Agroéconomie, ENSA, Montpellier, 220 p. https://agritrop.cirad.fr/ 300726/.

Bonnefond P, Couty P. 1988. Sénégal: passé et avenir d'une crise agricole. Revue Tiers-Monde 29(114): 319-340. https://doi.org/ 10.3406/tiers.1988.3542.

Camagni R. 2013. Regional competitiveness and territorial capital: A conceptual approach and empirical evidence from European Union. Regional Studies 47(9): 1383-1402. http://dx.doi.org/10.1080/ 00343404.2012 .681640$.

Cissé S, Diouf M. 2015. Lier la demande et l'offre de conseil agricole autour des grands barrages. Le cas de l'Anambé au Sénégal. Global Water Initiative. Rapport, 53 p. https://pubs.iied.org/fr/g03999.
Diouf MM. 2014. Un système officiel de commercialisation pour le riz local sénégalais. La voix de la vallée, 2 p. https://lavoixdela vallee.wordpress.com/2014/01/24/commercialisation-le-riz-localsenegalais/.

Fall AA. 2015. Synthèse des études sur l'état des lieux chaine de valeur riz au Sénégal. VECO, 55 p. https://www.inter-reseaux.org/ wp-content/uploads/rapport_final_etude_etat_des_lieux_filiere_ri Z_au_senegal_pdf.

FARM. 2018. Contractualiser avec les agriculteurs en Afrique. Groupe de travail contractualisation. Rapport final, 57 p. http:// www.fondation-farm.org/zoe/doc/rapportgtpoursite.pdf.

Faure G, Chiffoleau Y, Goulet F, Temple L, Touzard JM. 2018. Innovation et développement dans les systèmes agricoles et alimentaires. Versailles, France : Éditions Quae, 259 p. https:// www.quae.com/produit/1518/9782759228133/innovation-et-deve loppement-dans-les-systemes-agricoles-et-alimentaires.

Gabas JJ, Ribier V, Nubukpo K. 2019. Quelle place pour l'agriculture dans les plans émergence des gouvernements africains? In : Brot J (coord). L'émergence en question Marqueurs et dynamiques du développement. Les Cahiers de l'Association Tiers-Monde $\mathrm{n}^{\mathrm{0}} 34$. XXXIV journées sur le développement, pp.47-54. http://www. mondesendeveloppement.eu/medias/files/cahier-atm-34-2020.pdf.

GIZ (Agence allemande de coopération internationale). 2014. Manuel d'agriculture contractuelle. Guide pratique de mise en relation entre les petits producteurs/productrices et les entreprises acheteuses à travers l'innovation de modèles d'affaires. Bonn: GIZ, 116 p. https://www.snrd-africa.net/wp-content/uploads/2019/ 07/Manuel-dagriculture-contractuelle-Vol1.pdf.

Hazell P, Poulton C, Wiggins S, Dorward A. 2006. "The Future of Small Farms: Synthesis Paper", basé sur l'atelier de recherche organisé par l'IFPRI, ODI et Imperial College, Wye, 301 p. https:// documents 1.worldbank.org/curated/en/711841491218973857/ text/113954-WP-FRENCH-PUBLIC-Abstract-sent.txt.

Haussaire M. 2019. La fabrique de l'État sous régime d'aide. Construction d'une administration extravertie au Sénégal. Thèse de doctorat Science politique, Université de Lille, France, 511 p. https://hal.archives-ouvertes.fr/tel-02486354/document.

Hofmann A, Papazian V. 2020. L'irrigation : un outil pour la sécurité alimentaire et le développement économique des territoires. In: Bouarfa S, Brelle F, Coulon C (coord.). Quelles agricultures irriguées demain? Répondre aux enjeux de sécurité alimentaire et $d u$ développement durable. Versailles, France: Éditions Quae, pp. 47-57. https://www.quae.com/produit/1614/9782759231331/ quelles-agricultures-irriguees-demain.

Inter-réseaux, Laboratoire d'Analyse Régionale et d'Expertise Sociale (LARES). 2019. Rôle des interprofessions dans l'alimentation des marchés urbains. Synthèse de la capitalisation sur les interprofessions. Rapport, 25 p. https://www.interreseaux.org/wp-content/uploads/interprofessions_rapport_capi talisation_vf.pdf.

Lacquement ${ }^{-}$G, Chevalier P. 2016. Le programme européen LEADER en Europe centrale: apprentissage et pratiques de la gouvernance locale dans les campagnes post-socialistes. In: Lacquement G, Queva C, eds. Développement local et innovations sociales en Europe. Norois, 241, pp.67-83. https://doi.org/ 10.4000/geocarrefour. 15757.

Lazega E. 2014. Réseaux sociaux et structures relationnelles. Que sais-je ?, 128 p. https://doi.org/10.3917/puf.lazeg.2014.01.

Mendez del Villar P. 2019. Vers un renouveau de la riziculture pluviale au Sénégal. Le Blog Willagri, 5 p. https://www.willagri. com/2019/06/24/vers-un-renouveau-de-la-riziculture-pluviale-ausenegal/. 
Minot N. 2011. Contract farming in sub-Saharan Africa: Opportunities and challenges. In: Smallholder-led agricultural commercialization and poverty reduction: How to achieve it? Kigali (Rwanda): International Food Policy Research Institute, 30 p. http://fsg.afre.msu.edu/aamp/Kigali\%20Conference/Minot Contract_farming_(AAMP\%20Kigali).pdf.

Naudet JD. 1999. Trouver des problèmes aux solutions, vingt ans d'aide au Sahel. Paris (France): OCDE, Club du Sahel, 341 p.
Prowse M. 2013. L'agriculture sous contrat dans les pays en voie de développement, une revue de littérature. Paris (France): Agence française de développement, À Savoir n ${ }^{\circ} 12,56$ p. https://www.afd.fr/ sites/afd/files/imported-files/12-A-Savoir.pdf.

Vicente J, Balland P, Crespo J. 2018. Les fondements micro du changement structurel régional: Que nous enseignent vingt-cinq ans de proximités? Revue d'Économie Régionale \& Urbaine: 1013-1040. https://doi.org/10.3917/reru.185.1013.

Citation de l'article : Touré JMF, Chevalier P, Bourgoin J. 2021. Ancrage territorial du modèle contractuel rizicole sénégalais : une perspective géographique et relationnelle. Cah. Agric. 30: 48. 